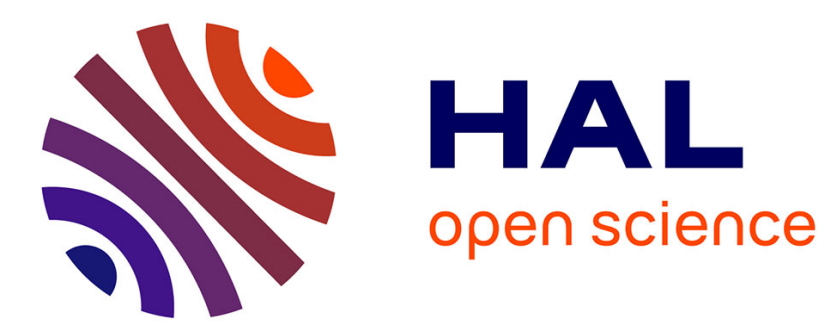

\title{
Transparent Collaborative haptic simulation
}

K. Tourbah, Hichem Arioui, Nicolas Seguy, Abderrahmane Kheddar

\section{To cite this version:}

K. Tourbah, Hichem Arioui, Nicolas Seguy, Abderrahmane Kheddar. Transparent Collaborative haptic simulation. IEEE Conference on Control Application (CCA 2006), 2006, Munchen, Germany. pp.187-192, 10.1109/CCA.2006.285890 . hal-00342996

\section{HAL Id: hal-00342996 \\ https://hal.science/hal-00342996}

Submitted on 29 May 2012

HAL is a multi-disciplinary open access archive for the deposit and dissemination of scientific research documents, whether they are published or not. The documents may come from teaching and research institutions in France or abroad, or from public or private research centers.
L'archive ouverte pluridisciplinaire HAL, est destinée au dépôt et à la diffusion de documents scientifiques de niveau recherche, publiés ou non, émanant des établissements d'enseignement et de recherche français ou étrangers, des laboratoires publics ou privés. 


\title{
Transparent Collaborative Haptic Simulation
}

\author{
K. Tourbah, H. Arioui, N. Séguy and A. Kheddar
}

\begin{abstract}
This paper deals with the problem of transparency (or fidelity) in force feedback systems or more particularly for haptics simulation under time delay transmission. The haptics rendering transparency is all the more difficult in distributed collaboration between distant users because it is necessary also to make feel faithfully the mutual interactions.

The idea suggested in this article gives an original solution to overcome this lack of transparency in haptic shared collaborations on the network using a principle called the "Latency Envelope" (LE). One advantage of this implementation is the anticipation of the contact between users and virtual objects. The delay can then be partially or completely compensated from the operator perception point of view. Also it does not affect at all the stability of haptics simulation (carried out using well known control laws).
\end{abstract}

\section{INTRODUCTION}

The collaboration haptics systems sharing a unique virtual environment, via a network have multi fields problems. Three main axes treated in literature in domain of force feedback from the most approached in literature in the delayed force feedback systems are: the control/command aspects of the mecatronic systems including the stabilization of the force feedback, the dynamic simulation within the virtual environment (mutual contact, collision detection, update of the virtual scenes...) and the network architecture used to set up the simulation (resources management, etc.).

In Teleoperation, the undesirable effect of the delay on stability was shown in 1965 by Ferrell [1]. An elegant solution is the one proposed by Anderson and Spong in [2] based on a quadripolar description of teleoperators (human operator, device master/slave, controllers and the slave environment). The approach recommended by Anderson and al [2] consists in imposing a perfectly passive line of transmission. It is then enough to determine the control laws which realize this passivity condition, knowing that the interconnection of passive elements leads to an unconditionally stable system. Results obtained with this command showed that the force feedback is well stabilized whatever size of the constant delay transmission is. However one can quickly note that the transparency in terms of follow-up velocity and the force feedback is somehow corrupted proportionally to the transmission delay size.

This approach was also used in more simple aspect by Niemeyer and Slotine called "wave variables" [3],[4],[5]. An

This work was supported by the Network of Excellence (NoE) INTUITION on Virtual Reality Reaserche Area.

All Authors are with IBISC CNRS FRE-2873 Laboratory, Evry Val d'Essonne University, CE 1455 Courcouronnes, 91025 Evry Cedex, France. Corresponding author: hichem.ariouidibisc.fr extension of this approach in the case of variable transmission delay was proposed by the same researchers [6] but unfortunately the approach remains too passive and does not allow the improvement of the force feedback fidelity. In the same way, other methods use adaptation based-passivity or robust control such us the work of Yokokodji and al [7],[8], Hannaford and al [9], etc. A second group of researchers adopted methods based on prediction, using for example device or delay behaviour models. In this case we can quote references of Saghir [10] and Arioui [11]. In this last paper [11], the author adapted the idea of the Smith predictor [12] without needing any information about the size and fluctuation of the delay, but only the model of the master device is necessary. Another advantage of this approach is that it can easily extend it to the case of the variable delay. Very simple to use, this approach allows an acceptable transparency except for the one related to the delay itself. At the same time, it still suffers from the robustness problems due to the imperfect prediction of the model. It uses, indeed, a master device based considered as linear.

In the haptic domain, we can witness in the past five years an increasing interest in collaboration systems using distributed virtual environments. The use of such systems is indeed largely advantageous in several fields (telesurgery, remote assistance, concurrent engineering, etc.) This works focus on the network aspects or the dynamic response in distributed environments to the detriment of control aspect like stabilization or transparency. The two remaining research axes (dynamic simulation and network architecture) will be slightly evoked in this paper. The interested readers can refer to the following references: Srinivasan and Basdogan [16],[17].

The article is organized as follows: in a first time, a simple model of the haptic device used during the experiments is described. The second part, discusses the choice of the method used to overcome the transmission delay problem and generalization to the cases of distant multi-users. In the third part, is treated the transparency problem and an original solution is proposed. At the end, simulation and experimental results are given.

\section{MODELLING OF THE HAPTIC SIMULATION}

The haptic interface used for the experimentation is a PHANToM (OMNI. This device is known for its lightness (apparent mass $m=45 \mathrm{~g}$ ) and very low viscous friction. For this reason, we simplify the device modelling (considered as a mass point) and give a first order linear model in each direction [11]. The device linearization was the object 


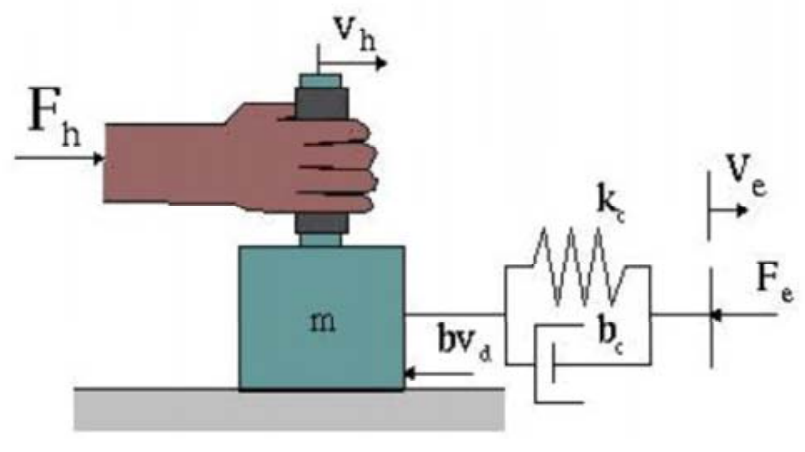

Fig. 1. Model of a linear haptic device with 1 DOF

of several researches like Salisbury's work [13] which was at the origin of interface design as well as the work of Cavusoglu and al [14].

The linear model of a haptic interface with one degree of freedom, figure (1), is given as follows:

$$
F_{h}(t)-F_{e}(t)=m \cdot \ddot{x}_{m}+b \cdot \dot{x}_{m}
$$

Where: $F_{h}$ and $F_{e}$ are the forces applied respectively by the human operator and the virtual environment on the device. $x_{m}, \dot{x}_{m}$ and $\ddot{x}_{m}$ represent respectively the position, speed and acceleration of the haptic device. $m$ and $b$ are the physical parameters (mass and friction) of the arm.

\section{A. Stabilization of the haptics simulation in the presence of the transmission delay}

As stated in the introduction, we chose the model based approach proposed in [11]. It suites to the type of the used arm and can easily be extended to the case of variable delay.

The dynamic equation from the operator $(i)$ point of view, in the presence of the delay (figure 2), is given as follows:

$$
F_{h i}(t)-F_{e i}(t-\tau(t))=m_{i} \cdot \ddot{x}_{m i}+b_{i} \cdot \dot{x}_{m i}
$$

$\tau(t)$ represent the variable transmission delay.

The use of "model-based control" scheme ${ }^{1}$ leads then to the new equation:

$$
F_{h i}(t-\tau(t))-F_{e i}(t)=m_{i} \cdot \ddot{x}_{m i}+b_{i} \cdot \dot{x}_{m i}
$$

Where only the refrence entry of the overall system (force of the operator, in our case) is delayed by $\tau(t)$, which is equivalent to the system of equation (1). Of course this change does not affect at all the stability of the initial complete stable system $(\tau(t)=0)$.

\footnotetext{
${ }^{1}$ This control scheme is giving by using a model prediction above and after the transmission channel to overcome delayed environment force $\left(F_{e}\right)$ effect and to replace it by pseudo command without delay. This scheme saves the force perception but eliminates easily the instability problem.
}

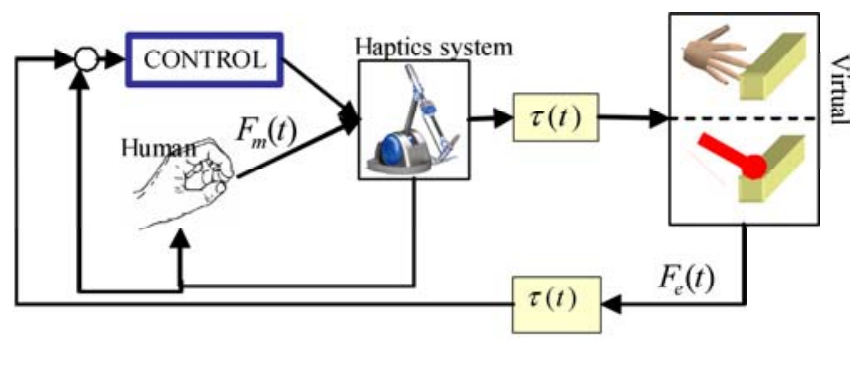

Fig. 2. Simplistic diagram of a haptic simulation with variable time delay in both direction

\section{B. Case of several distant operators}

The control scheme mentioned above is easily applicable to the collaborative simulation case (several distant operators). In this case, the used interfaces must have physical characteristics similar to those of the PHANToM DeskTop or OMNI, i.e. arms with low inertia and friction or must have linear associated models.

\section{HAPTIC COLLABORATION AND TRANSPARENCY}

\section{A. Haptic collaboration architecture}

We developed a client/server architecture based on an intelligent server where the processing power is shared between the clients as well as with the server. This server will manage all the resources of the simulation. It is the mediator between all the clients (operators) and its main task is, among others, to optimize the communication and resources use between the clients whatever the situation is. In order to operate properly the server to operate properly needs tools. We designed and developed tools that help the server to have a better knowledge of the virtual scene and thus take the best decision according to the situation. Among them, the one we named: "Latency Envelope" (LE).

\section{B. Transparency problem and proposed solution}

In haptic simulation, we often have the problem of transparency versus stability. A compromise must usually found. In our case, the intelligent server, with the help of the "LE", solves the problem of the visual-haptic fidelity, without affecting the stability which can easily be solved in an automatic way using classic control techniques. A similar principle to the latency envelope was evoked in Teleoperation by Chong and al in [19]. The envelope has a static form and implemented for multi-operators force feedhack. The latency envelope "LE" is used for motion prediction of the haptic feedback device in the virtual environment. This prediction is based on the velocity (or also the acceleration) of the manipulated objects as well as on the communication time delay. In practice, the LE has the same form as the original object with its main dimension increased with the calculated I.F size. The new created object is capable to 
make a good position prediction (see figure 3 ) of the haptic feedhack before the inevitable contact. Thus the LE allows to compensate the latency (partially or completely depending to the equation parameters) and to save the initial stability and interactive haptic feedback as possible. Other parameters are to be taken into account in the size calculation such as the nature of the link between the server and the client, the nature of the network used (dedicated line or not), haptic device position, velocity and direction of the "LE".

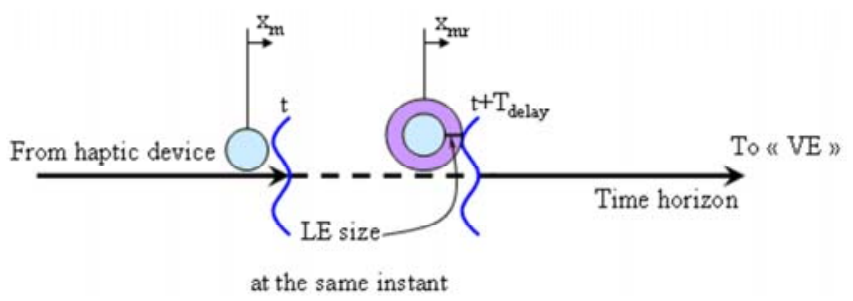

Fig. 3. Latency Envelope prediction motion principle

One of possible simple relation optimizing the LE size (SLE) is given as:

$$
S_{L E}=\frac{T_{\text {delay }} \cdot\left(K_{1}+\dot{x}_{v o}\right)}{K_{2}}
$$

with

- $T_{\text {delay }}$ is equal to the total time of the data processing, thus equal to the transmission time delay plus the time of the local processing.

- $\dot{x}_{v o}$ is the velocity of the handled virtual object (representation position haptic device).

- $K_{1}$ and $K_{2}$ are positive factors, to be synthesized according to the physical parameters of the simulation.

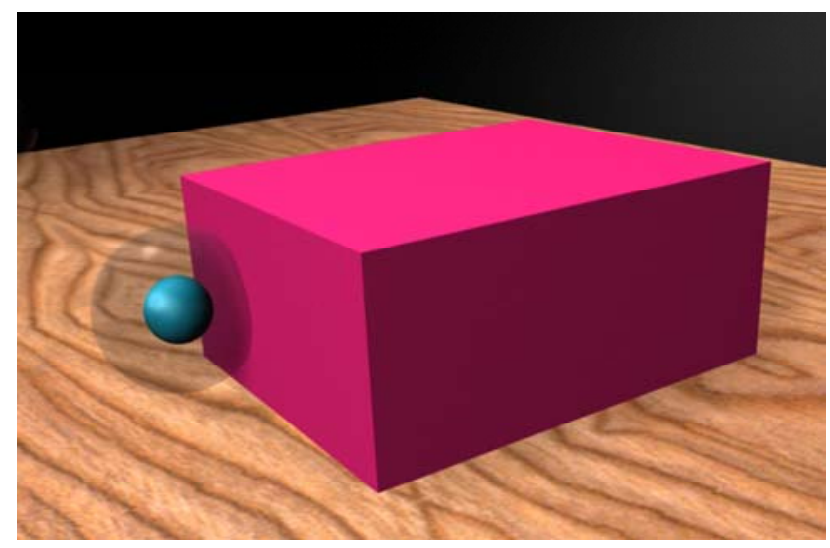

Fig. 4. Contact of a one client with a static object

Thus, if the total transmission delay $\left(T_{\text {delay }}\right)$ is zero, the I.F size is null and if the object's velocity is zero, the I.F. size is equal to the total data processing delay. Indeed, if the velocity of the virtual object is zero, it means that the client object is stationary and thus if another object has suddenly interacts with him, the client object is the same subject to the processing data time. But, if $T_{\text {delay }}$ is zero then the LF. size has no more reason to be, since the data is processed instantaneously by the client (see figure 4). Thus, there are no more visual-haptic fidelity problems due to time delay or even of stability problems which is impossible with the technology we currently use. Only the client's objects have, by default, a I.H of variable size according to the criteria previously explained. In the virtual environment, the static objects such us wall never have LE's (figure 4) and the dynamic objects take the largest LE of the client with whom they interact (figure 5).

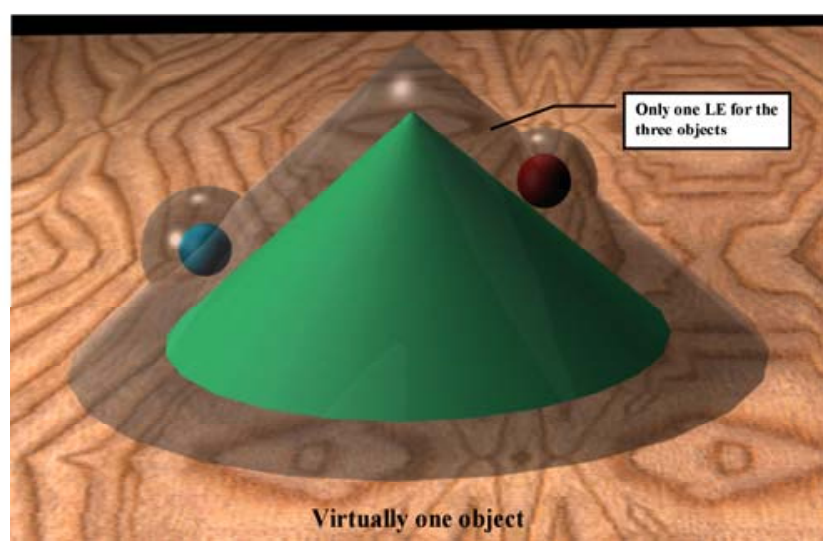

Fig. 5. Contact of two clients with a dynamic object

The surface ILE is used for collision detection, force generation and simulation dynamics until the virtual object collision. Once the surface of the LE collides with another object and the collision with the actual object is inevitable (due the velocity direction), the computational unit (server or another operator) sends immediately a force feedback command (i.e. calculated using penalty algorithm) to the operator. Due to this anticipation motion, the command arrives at the local machine roughly at the same time the contact happens. In spite of this time delay, the visual-haptic illusion is better and the collaborative haptic simulation is more transparent. Nevertheless, several problems arise: since the collisions detection is made using the envelope surface and since the force feedback is calculated with the distance of interpenetration, the force received by the operator can grow excessively. Furthermore, when the object leaves the contact, the collision detection is still made with the envelope and therefore the visual-haptic fidelity is affected. The operator still feels a force feedback while his object is not visually in contact anymore. For this reason, the calculation of the LE size was change and is now adaptive.

\section{Adaptive Latency Envelope "LE"}

In this case, theoretically the supposed LE will never cross the virtual wall, at least visually. Instead, at the contact instant, it narrows until completely disappearing when the main object will be itself collides with the same object (see figure 6). Once the contact "consumed" the I.F grows until reaching its "desired" or initial size. One second envelope, 

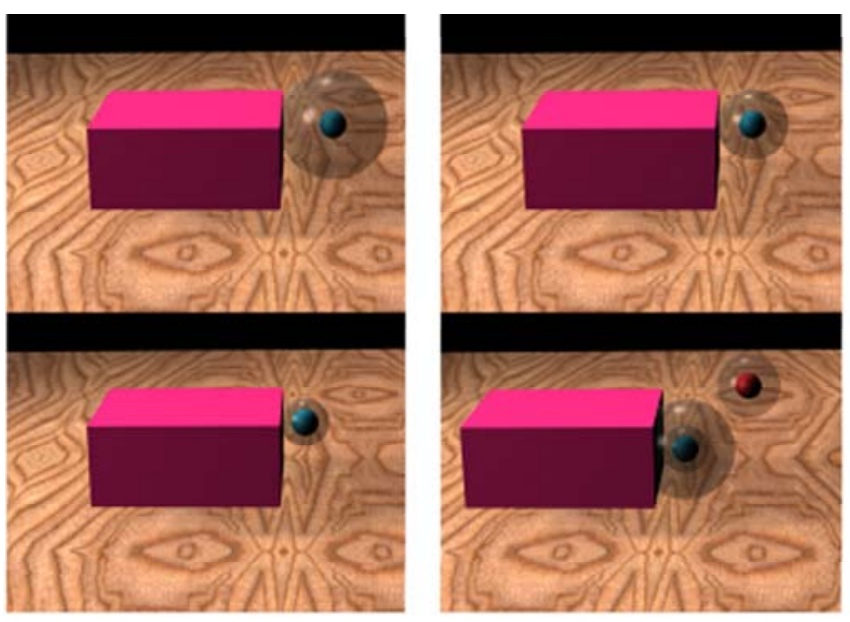

Fig. 6. Adaptive LE size

with the initial size, has to be present for a possible contact with second dynamic object during the contact with the first wall (see figure 6). Thus, the force feedback will never grow excessively and a threshold can be set to limit interpenetration distance. As the leaving of the contact is instantaneous it is impossible to predict the contact-end time. The visual-haptic fidelity is thus more accurate and the remaining problem is only due to the time delay. For an ideal visual-haptic perception, one must feel no forces at the instant when the object is visually no more in contact. To do so, as the contact ends, the local computer sends an order (force control) to its own haptic interface to stop the force feedback. It ignores all the delayed data received by the computational unit the envelope grows back to its initial size (according to the equation previously stated).

\section{SIMULATI(ON ANI EXPERIMENTATION RESULT}

\section{A. Simulations results}

Simulations have been done using MATLAB software in order to test the $\mathrm{LH}$ for the motion prediction of the haptic device. A one degree of freedom system has been modelled (as stated in the second section of this paper) in which an operator approaches his avatar toward a virtual wall. This last is modelled as spring-friction system. The simulated haptic interface is a linear device with an apparent mass $m=0.045 \mathrm{~kg}$ and have a coefficient of viscous friction $\mathrm{b}$ equal to $2 \mathrm{Ns} / \mathrm{m}$. The contact is calculated according to the interpenetration between the virtual object (representing the haptic interface within the virtual environment "VE") and the rigid virtual wall of stiffness $K_{e}=1000 \mathrm{~N} / \mathrm{m}$ (here, the viscous friction is ignored). The following figures (7 and 8) show the result obtained with a constant total delay of $1.5 \mathrm{sec}$ with and without the latency envelope "LE". A simple synthesis of the LE parameters $\left(K_{1}\right.$ and $\left.K_{2}\right)$ gives the following choice: $K_{1}=1$ and $K_{2}=30$. The time delay was set high in order to obtain a visually acceptable figure.

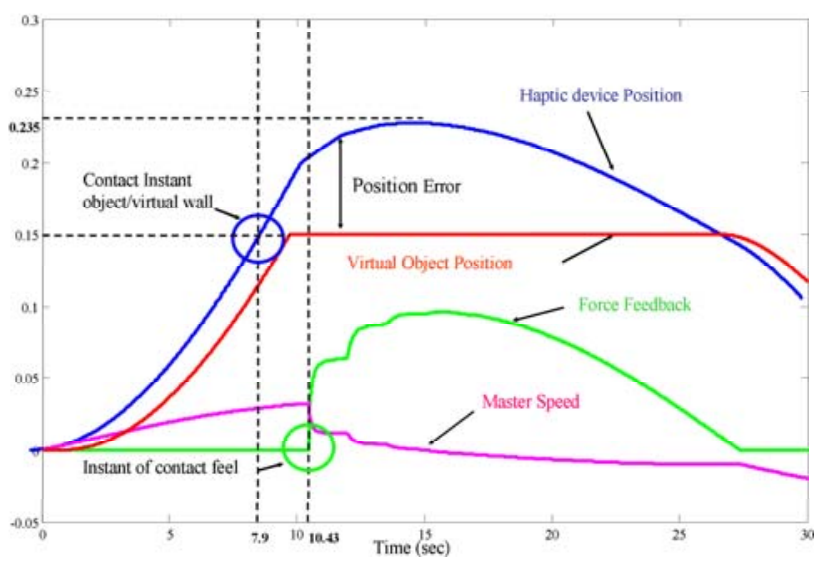

Fig. 7. Simulation of a haptic interaction with constant delay without LE

It shows the position tracking and the behaviour of the force feedback which remains stable when the operator interacts or comes into contact with the virtual wall. No additional disturbance is considered. In figure (7), case without LE prediction, when the contact occurs, the velocity of the haptic interface decrease quickly and the force feedback increases in order to establish a static balance during the contact. This phenomenon occurs after a response delay of $\tau_{1}=2.53 \mathrm{sec}$. The position error, about $0.085 \mathrm{~cm}$, which appears when the contact occurs, is thus impossible to avoid because of the transmission delay $\tau_{1}$.

We can also notice that, the use of latency envelope, the position error of contact is less compared to the case without LE algorithm. It decreases from 0.085 to $0.033 \mathrm{~cm}$. it clearly improves the visual-haptic illusion as well as the force feedhack fidelity (instant of contact sooner). The time between the visual contact and the perceived force feedback diminishes: $\tau_{1}$ goes from $2.53 \mathrm{sec}$ to $0.71 \mathrm{sec}$.

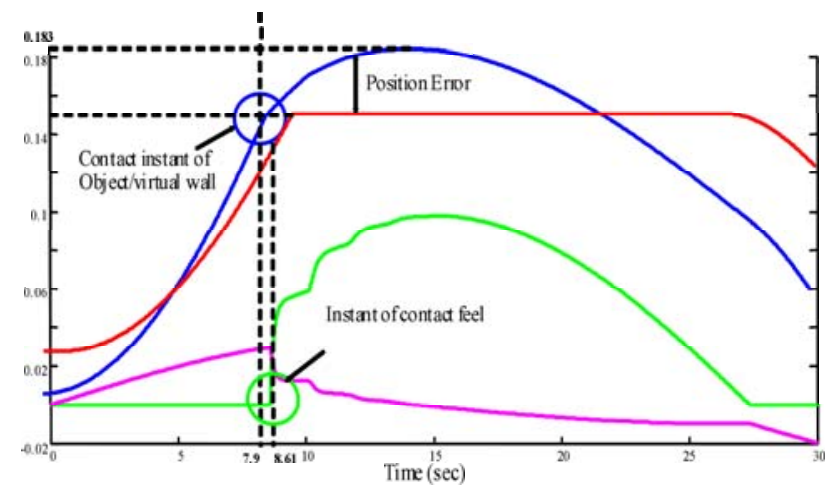

Fig. 8. Simulation of a haptic interaction with constant delay with LE and with $K_{2}=30$

At the contact time, the device position must follow as well as possible that of the virtual wall. It is thus necessary to minimize this error position while maintaining the force feedback throughout the contact time. 


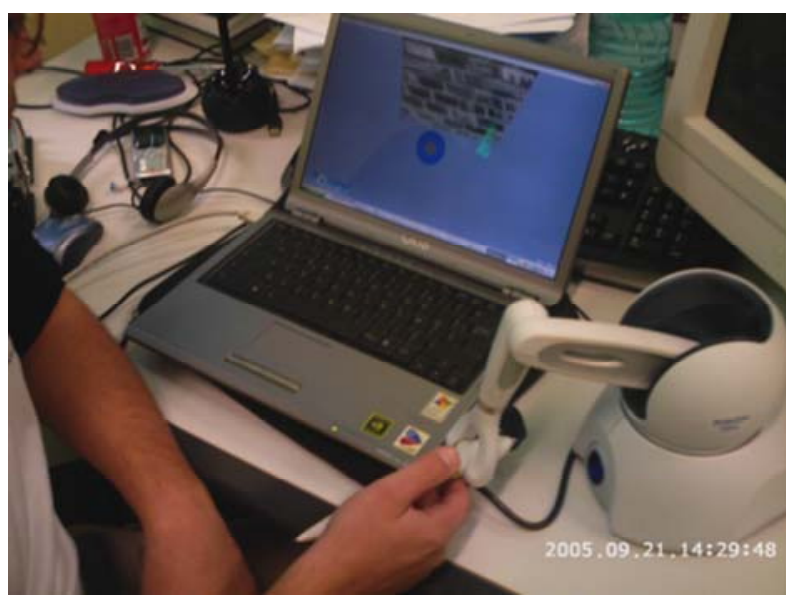

Fig. 9. Experimentation setup using an OMNI device and I-TOUCH Framework

\section{B. Experimental results}

Experimentations have been done using the PHANToM

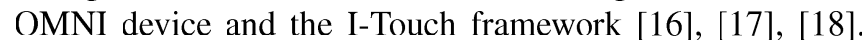
The global delay was simulated to be constant and equal to $1.5 \mathrm{sec}$. This choice is motivated to show clearly the force anticipation. An operator moves using the OMNI a virtual avatar against a virtual wall. Values of the avatar position, contact instant and force feedback have been recorded and plotted (figures 9 and 10).

We clearly can see that the stable force feedback sensed by the operator, at $2.8 \mathrm{sec}$, with the LE is anticipated compared to the one without, at $3.45 \mathrm{sec}$, and thus compensate a small amount of delay. This result can he sharply improved. The contact can be detected at $1.7 \mathrm{sec}$ by readjusting the size or coefficients relative to the Latency Envelope equation.

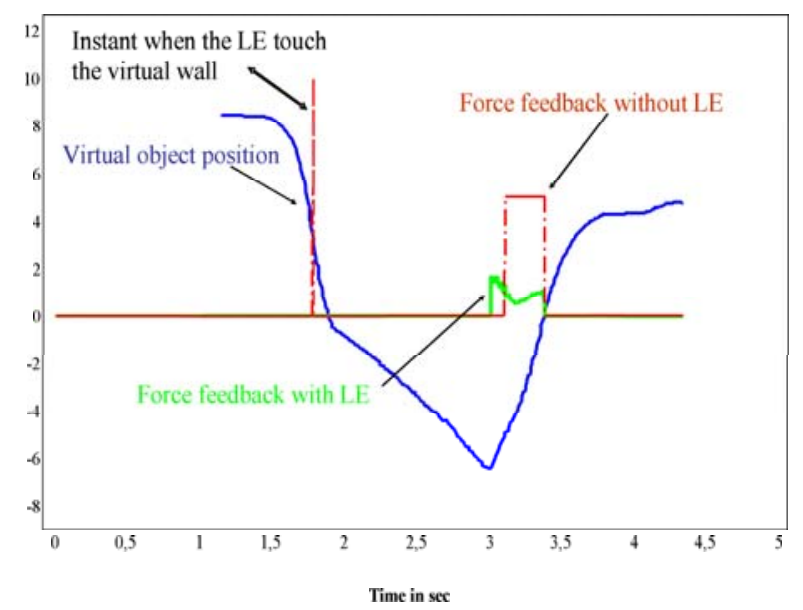

Fig. 10. Experimentation results

\section{CONCLUSIONS AND FUTURE WORKS}

In this paper we proposed an original solution for transparency issues directly related to the delay transmission present during a distributed haptic collaboration application. Simulations gave better results than experimentations because during simulation ideal conditions where used, i.e. constant time delay and of course no disturbances or noise due to the mechanical nature of the haptic device using for the experimentation. For a clear result and simplicity explanation we have chosen a constant delay case, in the same time we have obtain a acceptable simulation results in the case of variable delay. Future works concerns the implementation of a live distributed application i.e. between operators in different locations. The force feedback prediction procedure is quite simplistic and must include more parameters to suite to real conditions i.e. variable delay, unknown disturbances. Therefore, the I.H size equation has to be improved for example by the introduction a new factor linked to acceleration. Indeed, a sudden change in the acceleration modifies significantly the LE size and can thus corrupt the prediction. But, with the proposed adaptation, we can expect better result for non ideal situations.

\section{ACKNOWLEDGMENTS}

The authors gratefully acknowledge the very important contribution of Aurélien P()CHEVILLE and Benjamin BAYART to this work and experimentation.

\section{REFERENCES}

[1] W. R. Ferrell, Remote Manipulation with Transmission Delay, IEEE Transactions on Human Factors in Electronics, vol. 6, September 1965, pp 24-32

$\lceil 2\rceil$ R. Anderson and M. Spong, Bilateral control of Teleoperators with time delay, IEEE Transactions on Automatic Control, vol. 34, n5, pp. 494-501, May 1989.

[3] G. Niemeyer and J. I. Slotine, Stable Adaptive Teleoperation, IEEE Journal of Oceanic Engineering, vol. 16, pp. 152-162. 1991.

$\lceil 41$ G. Niemeyer and J. J. Slotine, Designing Force Reflecting Teleoperators with Time Delays to Appears as Virtual Tools, IEEE International Conference on Robotics and Automation, pp. 2212-2218, April 1997, Albuquerque, New Mexico.

$\lceil 5\rceil$ G. Niemeyer and J. J. Slotine, Using Wave Variables for system Analysis and Robot Control, IEEE International Conference on Robotics and Automation, pp. 1619-1625, April 1997, Albuquerque, New Mexico.

[6] G. Niemeyer and J. J. Slotine, Towards force-Reflecting Teleoperation over the Internet. IEEE International Conference on Robotics and Automation, pp. 1909-1915, May 1998, Leuven, Belgium.

17| Y. Yokokojhi, T. Imaida and T. Yoshikawa, Bilateral Teleoperation under Time-Varying Communication Delay, IEEE/RS.I International Conference Intelligent Robots and Systems, pp. 1854-1859, 1999.

$\lceil 81$ Y. Yokokojhi, T. Imaida and T. Yoshikawa, Bilateral Control with Energy Balance Monitoring Under Time-Varying Communication Delay, IEEE International Conference on Robotics and Automation, pp. 2434-2439, April 2000, San Francisco, CA.

[9] B. Hannaford and J. H. Ryu, Time Domain Passivity Control of Haptic Interfaces, IEEE Transactions on Rohotics and Automation, vol. 18, n1, pp. 1-10, February 2002.

$\lceil 10\rceil$ M. Saghir and W. J. Book, Wave-Based Teleoperation with Prediction, In Proceedings of the American Control Conference, pp. 4605-4611, June 2001, Arlington, VA.

[11] H. Arioui, A. Kheddar and S. Mammar, A model hased Controller for interactive force reflecting virtual environment under time delay, Journal of Intelligent and Robotic Systems -Theory and Applications -, June 2003, vol.37, n2, pp. 193-207.

$\lceil 12\rceil$ O. J. M. Smith, A Controller to overcome dead time, ISA Journal, vol. 6, n2, pp. 28-33, 1959. 
$\lceil 13\rceil$ J. K. Salisbury and M. A. Srinivasan, Phantom-Based Haptic Interaction with Virtual Objects, IEEE Computer Graphics and Applications, pp. 6-10, October 1997.

$\lceil 14\rceil$ M. C. Vavusoglu, D. Feygin and F. Tendick, A Critical Study of the Mechanical and Electrical Properties of the PHANToM Haptic Interface and Improvements for High-Performance Control, MIT Press - Presence, vol. 11, n6, pp. 555-568, December 2002.

$\lceil 15\rceil$ R. J. Adams. B. Hannaford and M. R. Moreyra, Stability and Performance of Haptic Displays, International Mechanical Engineering. Congress Exp., pp. 227-234, 1998, Anaheim, CA.

$\lceil 16\rceil$ A. Pocheville, A. Kheddar, and K. Yokoi, I-touch: A generic multimodal framework for industry virtual prototyping, TeXCRA, 2004.

$\lceil 17\rceil$ A. Pocheville and A. Kheddar, I-touch: a framework for computer haptics, IEEE/RSJ International Confernece on Intelligent Robots and Systems, Workshop, 2005.

[18] B. Bayart, A. Pocheville, and A. Kheddar, An adaptive haptic guidance software module for I-touch: example through an handwriting teaching simulation and a 3d maze, HAVE, 2005.

$\lceil 19\rceil$ N. Y. Chong, T. Kotoku, K. Ohba, K. Komoriya, N. Matsuhira and $\mathrm{K}$. Tanie, Remote coordinated controls in multiple telerobot cooperation, Proceedings of IEEE International Conference on Robotics and Automation, pp. 3138 -3143, 2000. 